\title{
Perioperative Management of Lactic Acidosis in End-Stage Liver Disease Patient
}

\author{
Alexander A. Vitin ${ }^{1 *}$, Leonard Azamfirei², Dana Tomescu³, John D. Lang ${ }^{1}$ \\ 1 Department of Anesthesiology \& Pain, Medicine University of Washington Medical Center, Seattle WA, USA \\ 2 University of Medicine and Pharmacy of Tîrgu Mureș, Romania \\ 3 "Carol Davila" University of Medicine and Pharmacy, Anesthesiology and Intensive Care Department 3, Fundeni \\ Clinical Institute, Bucharest, Romania
}

\begin{abstract}
Lactic acidosis (LA) in end-stage liver disease (ESLD) patients has been recognized as one of the most complicated clinical problems and is associated with increased morbidity and mortality. Multiple-organ failure, associated with advanced stages of cirrhosis, exacerbates dysfunction of numerous parts of lactate metabolism cycle, which manifests as increased lactate production and impaired clearance, leading to severe LA-induced acidemia. These problems become especially prominent in ESLD patients, that undergo partial hepatectomy and, particularly, liver transplantation. Perioperative management of LA and associated severe acidemia is an inseparable part of anesthesia, post-operative and critical care for this category of patients, presenting a wide variety of challenges. In this review, lactic acidosis applied pathophysiology, clinical implications for ESLD patients, diagnosis, role of intraoperative factors, such as anesthesia- and surgery-related, vasoactive agents impact, and also current treatment options and modalities have been discussed.
\end{abstract}

Keywords: lactic acidosis, end-stage liver disease, perioperative management, liver transplantation

Received: 10 March 2017 / Accepted: 28 April 2017

\section{INTRODUCTION}

End-stage liver disease (ESLD) is a multi-system illness, associated with variety of homeostasis derangements. Severe acid-base abnormalities, specifically increased anion gap metabolic acidosis, is considered as one of the most prominent and complex. Lactic acidosis (LA) has been recognized as the commonly encountered cause of metabolic acidosis [1]. The importance and prognostic value of this particular acid-base disturbance has been best illustrated by its association with mortality rates of $50 \%-56 \%$. It's been shown, that serum lactate levels above $4 \mathrm{mmol} / \mathrm{L}$, persistent after 24 hours, is associated with a survival of only $11 \%$ in critically ill patients [2].

In the ESLD patient, undergoing major surgery, such as liver resection or liver transplantation, development of LA becomes one of the major factors, associated with short- and long-term morbidity and mortality. Perioperative management of LA remains a complex and formidable task for anesthesiologist and critical care physician, with various aspects still being investigated.

\section{PATHOPHYSIOLOGY AND CLINICAL IMPLICATIONS}

There is no consensus as to what lactate concentration establishes the diagnosis of LA. For the time being, lactate concentrations $>5 \mathrm{mmol} / \mathrm{L}$ and a systemic $\mathrm{pH}<7.25$ serve as a defining point to that purpose [1]. Cohen and Woods [3] described causes of LA in two broad categories.

Type A: Clinical Evidence of Inadequate Tissue Oxygen Delivery. This category includes tissue hypoperfusion and reduced tissue oxygen delivery or utilization .

Type B: No Evidence of Inadequate Tissue Oxygen Delivery. This category has three subcategories, of which type $\mathrm{B} 1$ is associated with underlying disease, particularly, liver failure. B2 is associated with metabolites, drugs and toxins like acetaminophen, biguanides, cocaine, diethyl ether, epinephrine, norepinephrine, ethanol, and more. B3 type includes inborn errors of metabolism (congenital LA) e.g. glucose-6 phosphate dehydrogenase deficiency, Leigh's and Alpers diseases etc. 
Type B: No evidence of inadequate tissue oxygen delivery. This category has three subcategories, of which Type B 1 is associated with underlying disease, particularly, liver failure. This review will focus mostly on liver disease-associated Type B LA pathogenesis, clinical implications and management.

Lactate production and metabolism is well studied and described elsewhere [4]. The liver accounts for up to 50 to $70 \%$ of whole body lactate clearance, while the kidneys clear approximately $30-33 \%$ of lactate [5]. Chronic liver disease exacerbates hyperlactatemia in general, and especially in septic patients [6]. Hyperlactatemia and LA is common in fulminant liver failure, mostly due to both reduced clearance and increased production of lactate by the liver, and may serve as an important prognostic factor [5,7]. Long-standing LA by itself impairs lactate clearance even further, thus closing the vicious cycle. Acidemia contributes to decreased lactate removal by the liver; with severe hypoxia and acidemia converting the liver into essentially a net lactate-producing organ $[8,9]$.

Bleeding is commonly associated with hyperlactataemia in cirrhosis. Increased lactate production results from apparent tissue hypoperfusion (e.g. haemorrhagic shock) and from compromised cellular oxygen metabolism. In critically ill patients with severe liver dysfunction, cirrhosis and sepsis, severe acidemia is a marker of underlying organ dysfunction and buffer systems overload This may serve as a mortality predictor, independent of lactate concentration and LA presence [10]. Parameters such as $\mathrm{pH}, \mathrm{HCO}_{3}{ }^{-}$and base deficit have often have been shown to be consistently decreased in non-survivors in association with elevated lactate [11].

To diagnose LA as one of the primary acid-base disturbances, Stewart-Figgie methodology has been introduced. It suggests stepwise approach to quantitative acidosis analysis, in order to elucidate the lactic ion role in the ions balance.

Step one requires the calculation of strong ion difference, SID, using the formula:

$$
\operatorname{SID}_{\mathrm{a}}=\left[\mathrm{Na}^{+}\right]+\left[\mathrm{K}^{+}\right]+\left[\mathrm{Mg}^{2+}\right]+\left[\mathrm{Ca}^{2+}\right]-\left[\mathrm{Cl}^{-}\right]-[\text {lactate }-]
$$

SID represents the difference between the sum of concentrations of all strong cations and the sum of all strong anions.

Step two requires the calculation of effective SID, SIDe, which takes into account weak acids $\left(\mathrm{H}_{2} \mathrm{CO}_{3}\right.$, al- bumin and phosphate) (Figge modification):

SIDe $=2.46^{\star} 10-8 \times \mathrm{pCO} 2 /(10-\mathrm{pH})+[$ albumin $] \mathrm{x}$ $(0.123 \times \mathrm{pH}-0.631)+[\mathrm{phosphate}] \times \mathbf{( 0 . 3 0 9} \times \mathrm{pH}-0.469)$

Step three: calculation of the strong ion gap, SIG:

$$
\text { SIG }=\text { SIDa }- \text { SIDe }
$$

A positive SIG value indicates the presence of the unmeasured ions, such as sulfate, citrate, pyruvate, acetate, and likely substantial amounts of lactate, that are being responsible for increased anion gap(i.e. lactic acidosis) [12,13].

Deleterious effects of LA include decrease in cardiac contractility and cardiac output, and predisposition to cardiac arrhythmias, which can contribute to sudden death [14]. A reduction in intracellular $\mathrm{pH}$ can reduce cardiac contractility by competitive inhibition of $\mathrm{Ca}^{2+}$ binding to troponin. It decreases ATP production, possibly by inhibiting phosphofructokinase [15]. Although sympathetic stimulation accompanies the acidosis, responsiveness to both endogenous and infused catecholamines is attenuated. In vitro experiments have shown, that a reduction in external $\mathrm{pH}$ independent of any changes in intra-cellular $\mathrm{pH}$ reduces the binding of catecholamines to their correspondent receptors, attenuating the action of these components [16].

Metabolic acidosis, including LA, induces vascular smooth muscle relaxation via the opening of ATPsensitive potassium channels [17]. It also generates the expression of inducible nitric oxide synthetase in endothelium and vascular smooth muscle cells, causing overproduction of nitric oxide, thus exerts direct vasodilator effect on vascular beds [18].

\section{IMPACT OF ANESTHESIA AND SURGERY} (LIVER RESECTION AND LIVER TRANSPLANTATION)

The liver capacity for lactate clearance is directly dependent on adequate organ perfusion. Liver surgery (liver resection and, to lesser degree, radio-frequency tumor ablation (RFA) cause substantial fluctuations of hepatic blood flow (HBF), while liver transplantation (LT) causes the most dramatic changes in liver perfusion and all organ functions. It's been shown, that all general anesthesia techniques, regardless of drug choice, in the absence of surgical stimulation, reduce the HBF by about 30\% [19]. Surgery-related changes in $\mathrm{HBF}$, in addition to pre-existing organ perfusion problems, caused by cirrhosis, undoubtedly contribute to 
liver lactate production and clearance. In single case of severe LA during RFA, with no evidence of renal failure or peripheral perfusion problems, surgery-related liver dysfunction was found the only possible reason for LA development [20]. Several studies has shown that the blood lactate level was significantly elevated after occlusion of the liver vascular structures during partial hepatectomy $[21,22]$. In a relatively large clinical study involving 488 patients that underwent liver resection, $72 \%$ demonstrated elevated levels of lactate, which was associated with peak post-operative bilirubin, coagulopathy, renal dysfunction, diabetes, number of segments resected, blood loss and transfusion. It's been concluded that the initial post-operative lactate concentration is a useful predictor of outcome in liver resection patients [23]. Liver tissue hypoxia, that occurs during surgery, causes increased pyruvate conversion to lactate. This reaction is exacerbated by intra-operative stresses, such as blood loss, endogenous release of stress hormones and vasoactive agents administration [24]. Serum lactate can also be increased by transfusion of stored blood, which contains an ever-increasing concentration of lactate, depending on duration of storage [25].

Increased lactate levels in patients undergoing LT have been consistently observed. After induction of anesthesia, pre-induction lactate levels tend to rise. During the pre-anhepatic (dissection) phase, lactate accumulation occurs, most likely due to both HBF decrease and associated tissue hypoxia [26]. It's been found, that the rate of lactate accumulation during the pre-anhepatic phase was higher in patients with higher preoperative plasma bilirubin levels, thus more profound pre-operative liver dysfunction. [27].

In a patient with hemochromatosis, rapid development of severe decompensated LA during pre-anhepatic phase of liver transplantation (before vascular clamping) has been observed. The authors concluded that surgery and, perhaps, anesthesia-related factors most likely impaired perfusion and oxygenation of the native liver, resulting in decreased of liver lactic acid clearance [28].

The start of the anhepatic stage, during which time portal vascular structures are clamped and liver is removed from the body, halts any lactate processing and production in the liver. Lactate clearance will then be entirely kidney-dependent. Kidney dysfunction (such as hepatorenal syndrome or acute kidney injury) is common complication of ESLD, with an estimated prevalence of $30 \%$, and further exacerbates lactate clearance [29]. However, while acidosis significantly depresses hepatic uptake of lactate, it enhances renal lactate metabolism. The renal contribution to lactate removal thus increases from $16 \%$ at a pH of 7.45 to $44 \%$ at a $\mathrm{pH}$ of 6.75 . These changes account for approximately $50 \%$ of the hepatic loss of lactate metabolism [30]. Lactate removal by kidney mechanisms, is almost exclusively during the anhepatic stage, reperfusion, and a large portion of the neohepatic stage. This continues well into post-reperfusion and neohepatic stage.

Immediately after liver graft reperfusion, acidemia worsens substantially, oftentimes reaching the lowest $\mathrm{pH}$ value. However paradoxically, pre-reperfusion acidosis may be actually beneficial. It's been discovered, that naturally occurring acidosis protects against onset of cell death in many organs, including liver cells. Cell death in the $\mathrm{pH}$ paradox is not oxygen-dependent. Reperfusion at low pH $(<6.5)$ blocks increase of mitochondrial membrane permeability, which allows mitochondrial re-polarization and prevents cell death [31-33].

The ability of the recently reperfused liver graft to reintiate lactic acid processing depends on graft-recipient weight ratio (GRWR). It has been found, that this ratio is the only documented predictor of the lactate elimination ability after reperfusion in living-donor liver transplantation. Relatively larger grafts exhibit greater capacity for lactate elimination, whereas lactate levels may exceed the metabolic capacity of relatively small graft, resulting in lactate accumulation after reperfusion [34]. Lactate levels at the end of LT surgery, specifically threshold concentration of $5 \mathrm{mmol} / \mathrm{L}$, have been shown to have predictive value, and were associated with more than twofold -increased ICU length of stay and almost $100 \%$ 60-days mortality [35].

\section{EFFEcts of catecholamines AND VASO-}

\section{ACTIVE AGENTS ON LACTATE PRODUC- TION/ELIMINATION}

During LT, lactate production and elimination dynamics depend, in substantial degree, on changes of resulting catecholamine plasma concentrations. Use of vasoactive agents, essentially exogenic catecholamines, contribute to lactate production and elimination process in different ways. Specifically, norepinephrine has been shown to cause severe peripheral vasospasm and promote metabolic (lactic) acidosis [36]. In another 
study, lactate increased with a stable lactate/pyruvate $(\mathrm{L} / \mathrm{P})$ ratio with epinephrine and did not change with norepinephrine. It has been also demonstrated that epinephrine, but not norepinephrine increases lactate levels in animal experiment [37]. Physiologically, epinephrine enhances glycogenolysis with an increase in pyruvate production and ATP consumption by the $\mathrm{Na}^{+} / \mathrm{K}^{+}$pump, stimulating glycolysis and lactate production, thus increasing lactate level [38].

Phenylephrine, which is almost universally used during liver transplantation, has been shown to have no effect on lactate production and hepatic lactate utilization but has been associated with substantial decrease of blood lactate concentrations [39]. At least one animal study supports the lack of effect of dopamine and phenylephrine on LA during surgery [40]. The degree of phenylephrine contribution to lactate dynamics in LTs remain unclear and is probably worthy of further investigation.

Vasopressin has been shown to have a stimulation effect on lactate production by liver cells and adipose tissue in the septic model [41]. In our study, the lactate concentrations with vasopressin use were higher during the pre-reperfusion stage. This may be attributed to increased production of lactate by the gut and to impaired elimination of lactate by the native liver with additional vasopressin-related stimulation of lactate production. Difference in lactate concentrations between vasopressin and control groups substantially diminished toward the end of procedure, when vasopressin administration had already been discontinued, and the liver graft may have already resumed lactate processing [42].

Dopamine at low doses $(3-10 \mathrm{mcg} / \mathrm{kg} / \mathrm{min})$ significantly improve hepatic oxygenation and increased portal and total HBF during hypoxia. Dopamine infusion in sepsis models had no effect on either splanchnic lactate production or hepatic lactate utilization [43].

Regarding vasoactive agents, selection and dosage chosen during LT surgery maybe based on speed and magnitude of response. However, the ability of certain vasopressors to exacerbate lactic acidosis should definitely be considered during perioperative LA management $[44,45]$. This area remains open for investigation.

\section{MANAGEMENT OPTIONS}

The ultimate goal of perioperative management is to bring all parameters within physiological range. For the time being, no consensus exists as to when, to what extent, and how fast LA correction should be performed. In cases of severe acidemia, it's almost impossible to assess the actual role of lactate, contributing to the disturbance. One approach to LA treatment is to ignore the lactate altogether, and treat the associated acidemia. The problem with this approach is that, first, acidosis management may be inefficient without addressing the main cause; second, lactate itself exacerbates the liver and kidney lactate elimination insufficiency, rendering the treatment almost futile. Given the gravity of longstanding LA prognosis, active and early cause-oriented treatment is warranted. Lactate level-guided therapy, pursuing a goal of normalizing blood lactate levels (to $0.2 \mathrm{mEq} / \mathrm{L}$ ) has shown certain benefits [46]. It has become a widely accepted axiom, that hemodynamic optimization, aimed at lactate- processing organs (liver and kidneys) and tissues perfusion improvement, is a cornerstone of LA (and, in fact, any acidosis) management. However, excessive lactate production and inadequate elimination might exceed the corrective capacity of hemodynamic optimization alone. For those situations, other options are available.

\section{Sodium bicarbonate(SB)}

Sodium bicarbonate(SB) treatment, long time considered a cornerstone for any acidosis treatment, appears to be ineffective for LA correction. No benefit from SB therapy has been found in the management of LA in respect to clinical outcomes or mortality. SB failed to improve lactic acidosis, associated with malignancy . Sepsis- and phenphormin-associated LA proved to be resistant to SB as well [47]. Moreover, SB administration has been identified to be independently associated with higher mortality in several clinically relevant studies $[48,49]$. It's been demonstrated, that SB does not improve hemodynamics in critically ill patients with severe LA [50]. Studies also shown the ability of SB to increase plasma lactate concentration [51]. SB, while being inefficient in LA treatment, also isn't devoid of significant adverse effects. Complications of its use include intracellular acidification with severe impairment of cardiac function; depression of ionized $\mathrm{Ca}^{2+}$; volume overload; possible post-treatment metabolic intracellular acidification is more likely to occur when large amounts of bicarbonate are administered. If the circulation is adequate or only moderately impaired, bicarbonate administration might be beneficial, and actually improve acid-base parameters [52]. The administration of SB, therefore, should be individ- 
ualized. While SB administration may not be recommended in situations of circulatory collapse, following liver graft reperfusion, it may be beneficial in cases of severe acidemia due to chronic decompensated metabolic acidosis in patients with renal failure, complicating advanced liver disease.

\section{Dichloroacetate (DCA)}

DCA is considered to be an alternative treatment for metabolic, specifically lactic acidosis. It's not only neutralizing plasma $\mathrm{H}^{+}$, but also decreases blood and intracellular lactate concentrations by activating the mitochondrial pyruvate dehydrogenase, thus decreasing availability of pyruvate to form lactic acid. DCA, therefore, promotes intracellular homeostasis in myocardium and liver parenchyma during metabolic acidosis. Its been shown to be efficacious in a large population and over a broad range of lactate concentrations. The maximum lactate lowering effect of DCA is dosedependent but not time dependent [53]. The main advantage of DCA over SB is, therefore, its ability to prevent the intracellular acidosis development. It is also decreases dosage requirements for SB for the treatment of acidemia.

DCA is metabolized almost exclusively by the liver. Patients with cirrhosis display diminished hepatic pyruvate dehydrogenase activity, and also impaired plasma clearance of DCA. It has been observed that DCA administration causes a 50\% decrease in plasma lactic acid concentration during LT. Effect on lactate decrease during the first 70 minutes of anhepatic phase was also confirmed in animal experiments [54]. It is conceivable that using DCA alone may render SB either completely unnecessary or only used concomitantly at very low dosage. Giving its ability to correct both acidemia and lactic acid concentration, DCA should probably be reserved for those cases where rapid lactate accumulation along with acidemia occurs, and where large SB dosages would otherwise be required.

\section{THAM (Thromethamine)}

THAM, that buffers protons by virtue of the ammonia moiety, supplements the buffering capacity of the blood bicarbonate system, accepting a proton, generating bicarbonate and decreasing the partial pressure of carbon dioxide in arterial blood $\left(\mathrm{PaCO}_{2}\right)$. In contrast to $\mathrm{SB}$, it does not generate carbon dioxide. In fact, it might actually lower $\mathrm{PaCO}_{2}$ [55].
Administration of THAM in cases with mild LA in the ICU has been found equally effective to that of SB in improving extracellular acid-base parameters with no negative effects. [56].

The quantity of THAM required to increase serum concentrations of $\mathrm{HCO}_{3}{ }^{-}$is estimated by using the following formula:

\subsection{THAM requirement $($ in $\mathrm{ml})=$ lean body mass $(\mathrm{kg}) \times$ base deficit $(\mathrm{mEq} / \mathrm{l}) \times 1.1$,}

where base deficit = goal serum bicarbonate - actual serum bicarbonate [57]. In cases of impaired renal function (GFR $<30 \mathrm{ml} / \mathrm{min}$ ), THAM has a limited efficacy in protons removal. Significant retention of THAM in extracellular fluid compartments may lead to hyperosmolality. It has been demonstrated in experiments with animal LA model, that administration of THAM improved cardiac contractility, along with improvement in extracellular acid- base parameters [58]. THAM appears to be a useful alternative to SB in patients, that undergo LT, with LA causing severe acidemia, and at least partially preserved renal function. To date, however, its use in this particular setting has not yet been extensively investigated, and further prospective randomized controlled studies appear necessary.

\section{Place of hemodialysis and hemofiltration in intraop- erative lactic acidosis treatment}

Intraoperative use of continuous renal replacement therapy (CRRT), and hemofiltration during LT has been practiced for decades. Intraoperative CRRT has been used and has become a preferred option for acute correction of acid-base balance disturbances, electrolyte management, and intravascular volume adjustments during LT $[59,60]$. In one study, use of bicarbonate-based replacement fluid during continuous hemofiltration resulted in rapid resolution of the acidosis in $45 \%$ ( $n=200$ patients) patients with acute LA [61]. LA, that usually is developing during pre-anhepatic phase and worsening during anhepatic phase of LT, has become an important indication for intraoperative hemodialysis [28]. LA has been included as one of the non-renal indications for CRRT [62]. It's been found, that alkalinization by administered base during dialysis, augments lactic acid production by stimulating 6-phosphofructokinase. Nonetheless, dialysis can decrease hyperlactatemia and ameliorate acidemia, thus the use of hemodialysis and hemofiltration is advocated as treatment modalities for LA [63,64]. Several studies have looked into the efficacy of lactate removal 
by hemodialysis. It's been suggested, that lactate removal may participate in the correction of acidemia, observed during bicarbonate-based CRRT. Dialysis removes relatively small amount of lactate, compared to the produced quantity [65]. Lactate clearance, achieved by CRRT, has been found to be accounted for only< $3 \%$ of total lactate removal [66]. Nevertheless, dialysis therapy in patients with LA and impaired kidney function during LT appears to be an attractive option. Randomized controlled studies are necessary to prove the benefits of this treatment strategy, but may prove difficult due to its current rampant use.

\section{- CONCLUSIONS}

LA, commonly occurring intraoperatively in ESLD patients contributing greatly to perioperative morbidity and mortality. With the ultimate goal is to bring lactate levels close to physiological range (less than 0.2 $\mathrm{mEq} / \mathrm{L}$ ) cause-oriented, lactate-level guided perioperative management includes a number of options. Tissue perfusion optimization, which implies general hemodynamic stabiliztaion strategies aimed at lactate-processing organs (liver and kidney) functional improvement, balanced volume and blood loss management, and hemodynamic goal-oriented vasoactive agent administration. SB therapy appears the least effective option for LA treatment and should be reserved for moderate metabolic acidosis management to prevent the intracellular acidosis development. Dichloroacetate (DCA) and thromethamine (THAM) may be preferable options for LA intraoperative management, due to their efficacy in both lactate removal and acidemia correction with minimal side effects and absence of complications.

\section{DCONFLICT OF INTEREST}

None declared.

\section{口REERENCES}

1. Kreisberg RA. Lactate Homeostasis and Lactic Acidosis, Ann. of Int. Med. 1980;92: 227-237.

2. Gunnerson KJ1, Saul M, He S, Kellum JA. Lactate versus nonlactate metabolic acidosis: a retrospective outcome evaluation of critically ill patients Crit Care 2006; 10:R22.

3. Cohen RD, Woods HF. Clinical and biochemical aspects of lactic acidosis. Blackwell Scientific Publication, Oxford, 1976 pp.46-64.
4. Kraut J., Madias N. Lactic Acidosis N Engl J Med. 2014 Dec 11;371:2309-19.

5. Jeppesen JB, Mortensen C, Bendtsen F, Møller S. Lactate metabolism in chronic liver disease. Scand J Clin Lab Invest Scand J Clin Lab Invest. 2013;73:293-9.

6. Bakker J, Nijsten MW, Jansen TC. Clinical use of lactate monitoring in critically ill patients. Ann Intensive Care. 2013 May 10;3:12.

7. Murphy ND, Kodakat SK, Wendon JA, et al. Liver and intestinal lactate metabolism in patients with acute hepatic failure undergoing liver transplantation. Crit Care Med 2001; 29: 2111-8.

8. Oster JR, Perez GO. Acid-base disturbances in liver disease. J Hepatol 1986; 2: 299-306.

9. Walsh TS, Mclellan S, Mackenzie SJ, et al. Hyperlactatemia and pulmonary lactate production in patients with fulminant hepatic failure. Chest 1999; 116: 471-6.

10. Bernardi M, Predieri S. Disturbances of acid-base balance in cirrhosis: a neglected issue warranting further insights. Liver Int 2005;25:463-6.

11. Funk G., Doberer D., Kneidinger N., et al. Acid-base disturbances in critically ill patients with cirrhosis. Liver International 2007 ISSN 1478-3223 p.901-909.

12. Fencl V, Leith D. Stewart's quantitative acid-base chemistry: Applications in biology and medicine Resp. Physiol. 1993;91: 1-16.

13. Fencl V, Jabor A, Kazda A, Figge J. Diagnosis of metabolic acidbase disturbances in critically ill patients. Am J Respir Crit Care Med 2000;162:2246-51.

14. Orchard, C. H., Cingolani, H. E. Acidosis and arrhythmias in cardiac muscle. Cardiovasc. Res. 1994;28:1312-1319.

15. Halperin, M L, Cheema-Dhadli S, Halperin FA. et al. Rationale for the use of sodium bicarbonate in a patient with lactic acidosis due to a poor cardiac output. Nephron 1994;66: 258-261.

16. Huang, Y. G., Wong, K. C., Yip, W. H., et al. Cardiovascular responses to graded doses of 3 catecholamines during lactic and hydrochloric acidosis in dogs. Br. J. Anaesth. 1995;74: 583-590.

17. Ishizaka H, Kuo L. Acidosis-induced coronary arteriolar dilation is mediated by ATP-sensitive potassium channels in vascular smooth muscle. Circ Res. 1996;78:50-7.

18. Pedoto A, Caruso JE, Nandi J, et al. Acidosis stimulates nitric oxide production and lung damage in rats. Am J Respir Crit Care Med. 1999;159:397-402.

19. Gelman SI, Disturbances in Hepatic Blood Flow During Anesthesia and Surgery. Arch Surgery, 1976;111:881-893.

20. Shiokawa H, Akata T, Yoshino J, et al. Development of severe lactic acidosis during radio frequency ablation conducted for the treatment of hepatocellular carcinoma in a patient with liver cirrhosis; Masui. 2002;51:1263-7.

21. Yi Zou Y, Liu J, Liao Y, et al., Temporary hyperlactataemia 
Available online at: www.jccm.ro

during partial hepatectomy: Report of two cases Int J Surg Case Rep. 2015;6:285-288.

22. Pietsch U.C., Herrmann M.L., Uhlmann D., et al. Blood lactate and pyruvate levels in the perioperative period of liver resection with Pringle maneuver. Clin Hemorheol Microcirc. 2010;44:269-281.

23. Wiggans MG, Starkie T, Shahtahmassebi G,et al. Serum arterial lactate concentration predicts mortality and organ dysfunction following liver resection Periop. Medicine 2013;2:21-30.

24. Luchette FA, Jenkins WA, Friend LA et al. Hypoxia is not the sole cause of lactate production during shock. J Trauma 2002;52:415-419.

25. Uvizl R, Klementa B, Adamus M, et al. Biochemical changes in the patient's plasma after red blood cell transfusion. Signa Vitae 2011;6:64-71.

26. Koller J, Wieser C, Furtwangler W, et al. Orthotopic liver transplantation and perioperative lactate metabolism. Transplant Proc. 1991;23:1989-90.

27. Orii, R, SugawaraY; Hayashida M,et al; Peri-operative blood lactate levels in recipients of living-related liver transplantation Transpl. 2000;69:2124-2127.

28. Vitin A., Muczynski K, Bakthavatsalam R, et al. Treatment of severe lactic acidosis during the pre-anhepatic stage of liver transplant surgery with intraoperative hemodialysis. J of Clin Anesth. 2010;22:466-472.

29. Wu CC, Yeung LK, Tsai WS, et al. Incidence and factors predictive of acute renal failure in patients with advanced liver cirrhosis. Clin Nephrol 2006;65:28-33.

30. Yudkin J, Cohen RD: The contribution of the kidney to the removal of lactic acid load under normal and acidotic conditions Clin Sci Mol Med 1975;48:121-131.

31. Gores GJ,. Fleishman KE,. Dawson TE, et al. Extracellular acidosis delays onset of cell death in ATP depleted hepatocytes Am. J. Physiol. 1988;255:C315-C322.

32. Lemasters JJ, Nieminen AL, Qian T, Trost LC, et al. The mitochondrial permeability transition in cell death: a common mechanism in necrosis, apoptosis and autophagy. Biochim Biophys Acta. 1998;1366:177-96.

33. Fukazawa K, Vitin AA, Pretto EA Jr. Serum acidosis prior to reperfusion facilitates hemodynamic recovery following liver transplantation. J Anesth. 2016;30:80-8.

34. Jun JH, Kim GS, Na YR, et al. Analysis of Predictors for Lactate Elimination After Reperfusion in Recipients of Living-Donor Liver Transplantation. Transpl. Proc. 2014;46:709-711.

35. Jipa LN, Tomescu D, Droc G. The interrelation between arterial lactate levels and postoperative outcome following liver transplantation. Rom J of Anaesth Int Care 2014;21:106-112.

36. Levy B, Perez P, Perny J, et al. Comparison of norepinephrinedobutamine to epinephrine for hemodynamics, lactate metabolism, and organ function variables in cardiogenic shock. Crit Care Med. 2011;39:450-5.

37. Hargrove DM, Lang CH, Bagby GJ, Spitzer JJ Epinephrine-
The Journal of Critical Care Medicine 2017;3(2) • 61

induced increase in glucose turnover is diminished during sepsis. Metabolism 1989;38:1070-1076.

38. Levy B., Mansart A., Bollaert PE,et al.. Effects of epinephrine and norepinephrine on hemodynamics, oxidative metabolism, and organ energetics in endotoxemic rats. Intensive Care Med 2003;29:292-300.

39. Gregory JS, Bonfiglio MF, Dasta JF, et al. Experience with phenylephrine as a component of the pharmacological support of septic shock. Crit Care Med 1991;19:1395-1400.

40. Pascoe PJ, Ilkiw JE, Pypendop BH. Effects of increasing infusion rates of dopamine, dobutamine, epinephrine, and phenylephrine in healthy anesthetized cats. Am J Vet Res. 2006;67:1491-9.

41. Mandell MS, Katz JJ, Wachs $M$, et al. Circulatory pathophysiology and options in hemodynamic management during adult liver transplantation. Liver Transpl Surg 1997;3: 379-387.

42. Vitin AA, Martay K, Vater Y, et al. Effects of Vasoactive Agents on Blood Loss and Transfusion Requirements During PreReperfusion Stages of the Orthotopic Liver Transplantation. J Anesthe Clinic Res 2010;1:104-12.

43. Zaky A, Pretto EA Jr, Earle SA, et al. Hemodynamic and metabolic efficacy of dopamine versus norepinephrine in a brain-dead swine model. Liver Transpl. 2008;14:1266-72.

44. Kimmoun A, Novy E, Auchet $T$, et al. Hemodynamic consequences of severe lactic acidosis in shock states: from bench to bedside Critical Care 2015;19:175-188.

45. Levy B, Collin S, Sennoun N, et al. Vascular hyporesponsiveness to vasopressors in septic shock: from bench to bedside. Intensive Care Med. 2010;36:2019-29.

46. Jansen TC, van Bommel J, Schoonderbeek FJ, et al. Early lactateguided therapy in intensive care unit patients: a multicenter, open-label, randomized controlled trial. Am J Respir Crit Care Med. 2010;182:752-761.

47. D. S. Fraley, S. Adler, F. J. Bruns, et al, Stimulation of lactate production by administration of bicarbonate in a patient with a solid neoplasm and lactate acidosis The N Eng J of Med 1980;303:1100-1102.

48. Kim H J, Son YK, An WS., Effect of sodium bicarbonate administration on mortality in patients with lactic acidosis: a retrospective analysis PLOS One. 2013;8:e65283.

49. Wilson R.F., Spencer A. R., Tyburski J. et al. Bicarbonate therapy in severely acidotic trauma patients increases mortality. J of Trauma and Acute Care Surgery2013;74:45-50.

50. Cooper DJ, Walley KR, Wiggs BR, et al. Bicarbonate does not improve hemodynamics in critically ill patients who have lactic acidosis. A prospective, controlled clinical study. Ann Intern Med. 1990;112:492-8.

51. Fraley DS, Adler S, Bruns FJ, Zett B. Stimulation of lactate production by administration of bicarbonate in a patient with a solid neoplasm and lactic acidosis N Engl J Med. 1980;303:1100-2.

52. Kraut JA., Madias NE. Lactic Acidosis: Current Treatments and 
Future Directions. Am J Kidney Dis. 2016;68:473-482.

53. Shangraw RE.,Winter R., Robinson ST., et al. Amelioration of Lactic Acidosis with Dichloroacetate during Liver Transplantation in Humans. Anesth 1994;81:1127-38.

54. Shangraw RE, Fisher DM. Pharmacokinetics and pharmacodynamics of dichloroacetate in patients with cirrhosis. Clin Pharmacol Ther 1999;66:380-390.

55. Nahas GG1, Sutin KM, Fermon C, et al. Guidelines for the treatment of acidaemia with THAM. Drugs. 1998;55:191-224.

56. Hoste EA, Colpaert K, Vanholder RC, et al. Sodium bicarbonate versus THAM in ICU patients with mild metabolic acidosis. J Nephrol. 2005;18:303-307.

57. Kraut J A, Madias NE. Treatment of acute metabolic acidosis: a pathophysiologic approach Nat. Rev. Nephrol 2012;8:589-601.

58. Schotola H, Toischer K, Popov AF, et al. Mild metabolic acidosis impairs the beta-adrenergic response in isolated human failing myocardium. Crit Care. 2012;16:R153.

59. Townsend DR, Bagshaw SM, Jacka MJ, et al. Intraoperative renal support during liver transplantation. Liver Transpl
2009;15:73-78.

60. Parmar A, Bigam D, Meeberg G, et al. An evaluation of intraoperative renal support during liver transplantation: a matched cohort study. Blood Purif 2011;32:238-248.

61. Hilton, P. J., Taylor, L. G., Forni, L. G, Treacher, D. F. Bicarbonatebased haemofiltration in the management of acute renal failure with lactic acidosis. Q. J. Med 1998;91:279-283.

62. Schetz M. Non-renal indications for continuous renal replacement therapy. Kidney Int Suppl 1999;72:S88-S94.

63. De Corte W, Vuylsteke S, De Waele JJ, et al. Severe lactic acidosis in critically ill patients with acute kidney injury treated with renal replacement therapy. J Crit Care. 2014;29: 650-655.

64. Luft FC. Lactic acidosis update for critical care clinicians. J Am Soc Nephrol 2001;12(Suppl 17): S15-S19.

65. Levraut J, Ciebiera JP, Jambou P, et al. Effect of continuous venovenous hemofiltration with dialysis on lactate clearance in critically ill patients. Crit Care Med. 1997;25(1):58-62

66. Bellomo R., Bench-to-bedside review: Lactate and the kidney Crit Care. 2002;6:322-6. 